\title{
Probing Pocket Depth and Clinical Attachment Level between Non-Surgical and Surgical Periodontal Therapy in Chronic Periodontitis Patients: A Randomised Controlled Clinical Trial
}

\author{
Dr. Harish Kumar Shah, ${ }^{1}$ Dr. Shivalal Sharma, ${ }^{1}$ Dr. Khushboo Goel, ${ }^{1}$ \\ Dr. Sajeev Shrestha, ${ }^{1}$ Dr. Surya Raj Niraula ${ }^{2}$ \\ ${ }^{1}$ Department of Periodontology and Oral Implantology, College of Dental Surgery, BP Koirala Institute of Health Sciences, Dharan, Nepal; \\ ${ }^{2}$ Department of School of Public Health and Community Medicine, BPKIHS, Dharan, Nepal.
}

\begin{abstract}
Background: : Chronic periodontitis is one of the most common form of periodontal diseases which either require non-surgical periodontal therapy or open flap debridement-surgical therapy or both. To date, it is unclear as of how much changes occur after NSPT or OFD and which therapy provides the best outcome in chronic periodontitis having probing pocket depth $\geq 5-7 \mathrm{~mm}$.

Aim: The aim of this randomized controlled clinical trial was to evaluate the Probing Pocket Depth and Clinical Attachment Level between NSPT and OFD in chronic periodontitis patients.

Materials and Methods: A total of 52 healthy patients with PPD $\geq 5-7 \mathrm{~mm}$ were included in the present study. Half of the patients assigned for the NSPT and half in the OFD group. The PPD and CAL were measured at baseline, three and six months. Independent sample t-test was used to compare the change in mean PPD and CAL between NSPT and OFD group at three and six months, respectively.

Results: The difference in the mean decrease of PPD between NSPT and OFD group at three and six months were 0.15 mm $(\mathrm{P}<0.05)$ and 0.19 $\mathrm{mm}(\mathrm{P}<0.05)$, respectively. The difference in the mean gain of CAL between NSPT and OFD group at three and six months were 0.03 mm $(\mathrm{p}>0.05)$ and $0.12 \mathrm{~mm}(\mathrm{P}<0.05)$, respectively.

Conclusion: Substantial improvement in periodontal status occurred with both the therapies, however, significantly higher decrease in PPD and gain in CAL were seen with surgical therapy.

Keywords: Clinical attachment level; non-surgical periodontal therapy; probing pocket depth; surgical periodontal therapy.
\end{abstract}

\section{INTRODUCTION}

Chronic periodontitis has been defined as "an infectious disease resulting in inflammation within the supporting tissues of the teeth, progressive attachment loss, and bone loss." ${ }^{1}$ Almost $10-15 \%$ of the population worldwide is suffering from a severe form of periodontitis according to a report published in 2005 by the World Health Organization. ${ }^{2}$ In Nepal, $29 \%$ of aged 35-44 years are suffering from deep periodontal pockets. ${ }^{3}$ Chronic periodontitis demands periodontal therapies that include either non-surgical periodontal therapy (NSPT) or open flap debridement (OFD) surgical therapy or both to arrest the progression of a disease. ${ }^{4}$ To date, it is unclear as which therapy is superior for probing pocket depth (PPD) $\geq 5-7 \mathrm{~mm}$ and how much decrease of PPD and gain in clinical attachment level (CAL)

\section{Correspondence: \\ Dr. Harish Kumar Shah \\ Department of Periodontology and Oral Implantology, College of Dental Surgery, BP Koirala Institute of Health Sciences, Dharan, Nepal. \\ email: harishshah46@yahoo.com}

Citation

Shah HK, Sharma S, Goel K, Shrestha S, Niraula SR. Probing Pocket Depth and Clinical Attachment Level between Non-Surgical and Surgical Periodontal Therapy in Chronic Periodontitis Patients: A Randomized Controlled Clinical Trial. J Nepal Soc Perio Oral Implantol. 2018;2(2):40-4. occur after surgical and non-surgical periodontal therapies (NSPT). To the best of our knowledge, this study is the first of its kind in Nepal which has evaluated decrease in PPD and gain in CAL after periodontal therapies. The objective of this study is to evaluate the PPD and CAL between NSPT and open flap debridement (OFD) in chronic periodontitis.

\section{MATERIALS AND METHODS}

A randomised controlled clinical trial was carried out between May 2016 to January 2018, in the Department of Periodontology and Oral Implantology, College of Dental Surgery, B.P. Koirala Institute of Health Sciences, Dharan, Nepal. Ethical approval for the study was obtained from the Institutional Review Committee Dharan. All the patients visiting the out patient department (OPD) and fulfilling inclusion criteria were participants of this study. The inclusion criteria were patients with chronic periodontitis, age range between 30 to 55 years, having at least 20 natural teeth within both jaws, with PPD 5 to $7 \mathrm{~mm}$ in at least two or more sites of non-adjacent teeth. The exclusion criteria were patients with a known systemic disease, history of a known infective disease other than periodontitis, use of an antibiotic in the preceding three months, history of receiving treatment with any medications known to affect periodontal health, pregnant females, history of a receiving periodontal treatment within three months, smokers. 


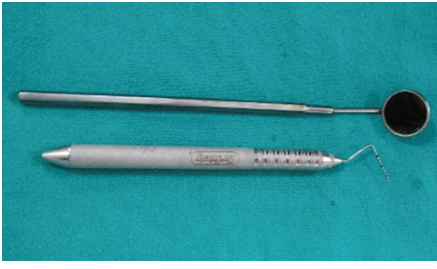
15 probe.
Figure 1a: Mouth mirror and UNC

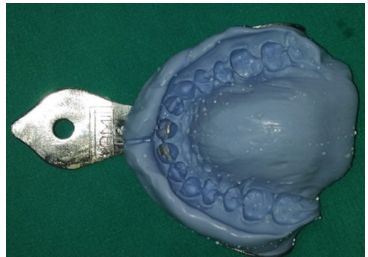

Figure 1b: Alginate impression material.
Patient recruitment: The patients diagnosed with chronic periodontitis meeting the inclusion criteria were selected. Prior to the initiation of this study, a written informed consent was obtained from each patient after explaining the nature of the study. All the selected patients underwent supra-gingival scaling. Two weeks following supra-gingival scaling, 52 patients of chronic periodontitis were enrolled in the study. The patients were divided into two groups; group I for NSPT and group II for OFD. The NSPT and OFD periodontal therapies were coded and sealed in the opaque envelope which were revealed only at the time of the procedure. All periodontal parameters were collected by the principal investigator.

Randomisation of patients was done by lottery method and this method was used for assigning the patient to two different treatment procedures (NSPT or OFD). One box which consisted of 52 sequentially numbered written paper slips in an opaque envelope. Twenty-six labeled paper slips for OFD and next 26 for NSPT. The patient was asked to pick one opaque envelope for his/her therapy on the day of treatment and other investigator performed both the NSPT and OFD who was not involved in data collection.

Periodontal Parameter measured: PPD was measured from gingival margin to the base periodontal pocket. Clinical attachment level (CAL) was measured from the cementoenamel junction to the base periodontal pocket. PPD and CAL were measured at baseline and at the interval of three and six months after periodontal therapy. For the fabrication of splint, an alginate impression was made (Figure 1b). The PPD and CAL were recorded manually using a University of North Carolina-15 (UNC-15) probe, mouth mirror and custom-made acrylic occlusal stent. The customised acrylic stent was fabricated with cold cure acrylic on a cast. A fixed groove was made with bur for reference. Custom-made acrylic occlusal stent with groove was used for precise alignment of the probe for measuring PPD and CAL in all the patient.

\section{Periodontal therapy:}

\section{Non-surgical periodontal therapy}

NSPT included supra and subgingival ultrasonic scaling and root planing with NSK (Varios 570 iPiezo engine) ultrasonic scaler and oral hygiene instructions. $0.2 \%$ chlorhexidine digluconate oral rinses for plaque suppression twice a day for two weeks was given after scaling and root planing (SRP). Patients were kept on two-weekly Follow-up till one month for supra-gingival scaling, initial clinical response evaluation, and oral hygiene reinforcement, then they were kept on monthly Follow-up for supra-gingival scaling and oral hygiene reinforcement over the entire duration of the study.

\section{Surgical therapy (OFD):}

One month prior to surgery all patients received phase I therapy (hygienic phase) which included supra and subgingival scaling and plaque control instructions. Patients exhibiting an O'Leary plaque index $<25 \%$, persistence of bleeding on probing, $\geq 5-7 \mathrm{~mm}$ PPD at the time of surgery were only included for OFD. ${ }^{5-6}$ It was done after proper extraoral asepsis with $5 \%$ povidone-iodine and $0.2 \%$ preprocedural rinse with chlorhexidine. Open flap debridement (Modified flap operation-Kirkland 1931) was done. ${ }^{7}$ After adequate local anaesthesia; sulcular incision was made on both the labial and the lingual aspects of the interdental area. The incisions were extended in a mesial and a distal direction, and mucoperiosteal flap was reflected. The defects were carefully debrided. Angular bony defects were curetted but no bone was removed. Extensive removal of non-inflamed tissues and intentional apical displacement of the gingival margin were avoided. Following the elimination of the pocket epithelium and granulation tissue from the inner surface of the flaps, these were replaced at their original position and complete wound closure was achieved with interrupted ligation with 4-0 silk suture (Figure 2). Tablet Ibuprofen $400 \mathrm{mg}$ three times a day ( 8 hourly) for three days as analgesic and chlorhexidine digluconate $0.2 \%$ rinse for plaque inhibition twice a day for two weeks were prescribed. Surgical therapy was done once and then patients were on Follow-up weekly for the next four weeks for professional
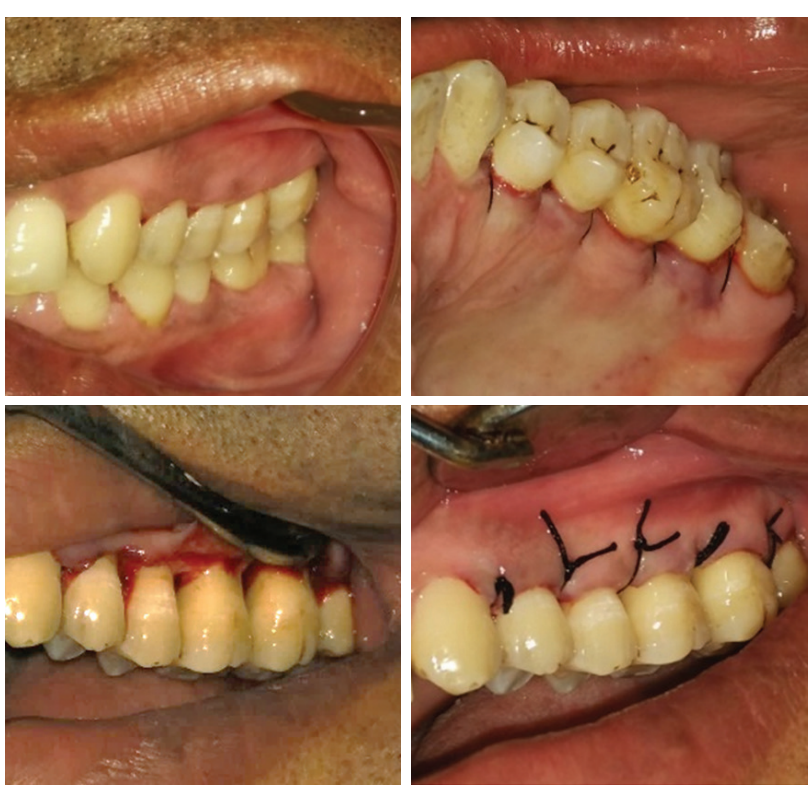

Figure 2: Open flap debridement-Surgical therapy. 
plaque control and then kept on monthly Follow-up over the entire duration of the study.

The data was entered in MS-Excel 2007 and was analysed by SPSS, version 11.5. Descriptive statistics (mean, standard deviation) were calculated of before and after periodontal therapy. Two independent sample t-test was used to compare change in mean PPD and change in mean CAL between OFD and NSPT groups. The probability of significance was set at $5 \%$ level.

\section{RESULTS}

This single centered randomised clinical trial was carried out to evaluate the change in probing pocket depth after NSPT and OFD in chronic periodontitis patients. A total of 52 patients (16 males and 36 females) with mean age 42.31 years were enrolled (Table 1). All the enrolled patients $(\mathrm{N}$ = 52) completed the trial and analysis of all patients were done as shown in the CONSORT diagram.

In the NSPT group (Table 2), the mean PPD at baseline, 3

Table 1: Age of the participants in years.

\begin{tabular}{|c|c|c|}
\hline Age & Mean & Standard deviation \\
\hline Number of Patients $(\mathrm{N})=52$ & 42.31 & 6.49 \\
\hline
\end{tabular}

and 6 months were $6.23 \mathrm{~mm}, 5.29 \mathrm{~mm}$ and $4.36 \mathrm{~mm}$, respectively and mean change from baseline to 3 months and baseline to 6 months were $0.94 \mathrm{~mm}$ and $1.87 \mathrm{~mm}$, respectively. Similarly, in the OFD group, the mean PPD at baseline, 3 and 6 months were $6.22 \mathrm{~mm}, 5.14 \mathrm{~mm}$ and $4.17 \mathrm{~mm}$, respectively and mean change from baseline to 3 months and baseline to 6 months were $1.07 \mathrm{~mm}$ and 2.04 $\mathrm{mm}$, respectively. The decrease of PPD at 6 months was greater than 3 months in both the groups. The difference in the mean decrease of PPD between NSPT and OFD group at 3 and 6 months were $0.15 \mathrm{~mm}(\mathrm{P}<0.05)$ and $0.19 \mathrm{~mm}(\mathrm{P}<0.05)$ and from baseline to 3 months and baseline to 6 months were $0.13 \mathrm{~mm}(\mathrm{P}<0.05)$ and $0.17 \mathrm{~mm}(\mathrm{P}<0.05)$, respectively.

Likewise, in the NSPT group (Table 3), the mean CAL at baseline, 3 and 6 months were $5.32 \mathrm{~mm}, 5.23 \mathrm{~mm}$ and $4.31 \mathrm{~mm}$, respectively and mean change from baseline to 3 months and baseline to 6 months were $0.09 \mathrm{~mm}$ and 1.00 $\mathrm{mm}$, respectively. However, in the OFD group, the mean CAL at baseline, 3 and 6 months were $5.25 \mathrm{~mm}, 5.19 \mathrm{~mm}$ and $4.19 \mathrm{~mm}$, respectively and mean gain from baseline to 3 months and baseline to 6 months were $0.06 \mathrm{~mm}$ and 1.06 $\mathrm{mm}$, respectively. The gain of CAL at 6 months was greater than 3 months in both the groups. The difference in the mean gain of CAL between NSPT and OFD group at 3 and 6 months were $0.03 \mathrm{~mm}(\mathrm{P} .>0.05)$ and $0.12 \mathrm{~mm}(\mathrm{P}<0.05)$ and from baseline to 3 months, and baseline to 6 months were $0.02 \mathrm{~mm}(\mathrm{p}>0.05)$, and $0.06 \mathrm{~mm}(\mathrm{P}<0.05)$, respectively.

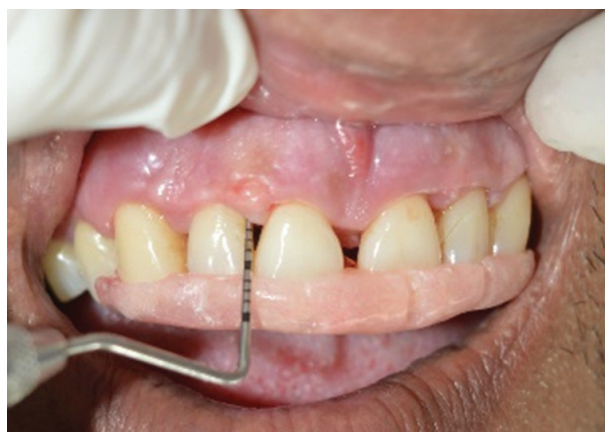

Figure 3: PPD and CAL recording with the stent and UNC-15 probe.

Table 2: PPD at baseline, 3 and 6 months after NSPT and OFD.

\begin{tabular}{|l|c|c|c|c|c|}
\hline \multicolumn{1}{|c|}{ PPD } & T0 & T3 & T6 & T0-T3 & T0-T6 \\
\hline NSPT $(\mathrm{n}=26)$ & $6.23 \pm 0.14$ & $5.29 \pm 0.18$ & $4.36 \pm 0.21$ & $0.94 \pm 0.16$ & $1.87 \pm 0.17$ \\
\hline Mean difference b/w NSPT and OFD & 0.01 & 0.15 & 0.19 & 0.13 \\
\hline OFD $(\mathrm{n}=26)$ & $6.22 \pm 0.15$ & $5.14 \pm 0.12$ & $4.17 \pm 0.13$ & $1.07 \pm 0.12$ & $2.04 \pm 0.13$ \\
\hline P value & 0.705 & $0.001 *$ & $<0.001 *$ & $0.001 *$ \\
\hline
\end{tabular}

$N=26$ (number of patients in each NSPT and OFD group),

TO = baseline, $T 3=3$ months, T6 = 6 months,

TO-T3 = difference between $(b / w)$ baseline and 3 months, T0-T6 = difference $b / w$ baseline and 6 months

Confidence interval $=95 \% \quad$ Independent sample $t$-test was used

The mean difference is significant at the 0.05 level.*. ( $P<0.05$ as significant)

Table 3: CAL at baseline, 3 and 6 months after NSPT and OFD.

\begin{tabular}{|l|c|c|c|c|c|}
\hline \multicolumn{1}{|c|}{ CAL } & T0 & T3 & T6 & T0-T3 & T0-T6 \\
\hline NSPT $(\mathrm{N}=26)$ & $5.32 \pm 0.15$ & $5.23 \pm 0.14$ & $4.31 \pm 0.16$ & $0.09 \pm 0.10$ & $1.00 \pm 0.10$ \\
\hline Mean difference b/w NSPT and OFD & 0.06 & 0.03 & 012 & 0.02 \\
\hline OFD $(\mathrm{N}=26)$ & $5.25 \pm 0.14$ & $5.19 \pm 0.12$ & $4.19 \pm 0.13$ & $0.06 \pm 0.83$ & $1.06 \pm 0.11$ \\
\hline P value & 0.114 & 0.310 & $0.003 *$ & 0.295 \\
\hline
\end{tabular}

$N=26$ (number of patients in each NSPT and OFD group)

TO = baseline, T3 = 3 months, T6 = 6 months,

TO-T3 = difference between $(b / w)$ baseline with 3 months, TO-T6 = difference $b / w$ baseline and 6 months

Confidence interval $=95 \% \quad$ Independent sample t-test was used

The mean difference is significant at the 0.05 level. $*$. $(P<0.05$ as significant) 


\section{CONSORT Diagram}

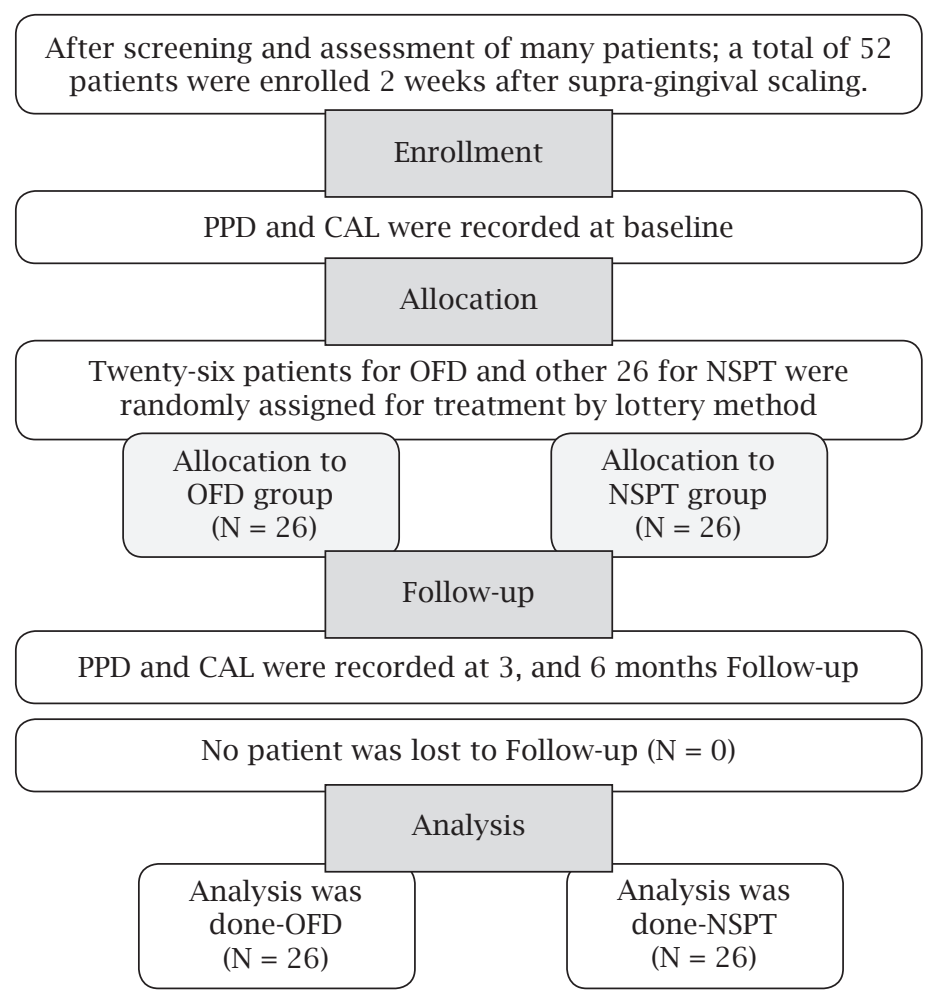

Figure 4: Participant enrollment, Allocation, Follow-up and Analysis.

\section{DISCUSSION}

The aim of periodontal therapy is to arrest the progression of periodontal disease and to get stable periodontium. In this study, baseline data were recorded two weeks following supra-gingival scaling in all patients to standardised the baseline measurement by reducing distortion in assessment and gingival inflammation. The customised stent with fixed groove was used for angulation control of the probe for measurement of probing pocket depth and clinical attachment level (Figure 3).

There was substantial improvement of periodontal status in terms of decrease in PPD and the gain of CAL from baseline to six months after NSPT and OFD. The PPD and CAL were recorded at three and six months in both NSPT and OFD group in the present study which is in accordance with studies done by Kim et al, Haffajee et al, and Badersten et al. ${ }^{8-10}$ Egelberg (1999) cited that three months post-therapy is a suitable interval for the primary evaluation of initial non-surgical therapy, even in areas with preliminary deep lesions. ${ }^{11}$ Rylander and Lindhe (2003) cited that healing after non-surgical therapy seemed to be complete after three to six months in those who use proper oral hygiene measures. ${ }^{12}$ According to Hall (2003), surgical therapy reevaluation should be performed approximately three months after the completion of surgical therapy and this evaluation is similar to non-surgical evaluation and assesses the success of surgical therapy. ${ }^{13}$ Histological evidence indicates that gain of clinical attachment after root planing does not represent new connective tissue attachment, but corresponds to a reduction of inflammation in the gingival connective tissue and the formation of a long junctional epithelium. ${ }^{14}$
Need for surgical therapy can be only assessed by completion of one month hygienic phase in which clinical severity of periodontitis reduced significantly. ${ }^{15}$ Surgical therapy was done following one month hygienic phase of periodontal therapy which agrees with Morrison et al. ${ }^{15}$ The consensus report from the American Academy of Periodontology World Workshop agreed that a four to six weeks interval was usually adequate to assess the initial response to therapy. ${ }^{16}$ In case of surgical therapy, Follow-up was done weekly for suture removal, polishing of teeth, supra-gingival scaling, initial response evaluation and plaque control reinforcement in accordance to Kaldahl et $\mathrm{al}^{6}$ and monthly for maintenance therapy over the entire duration of study. In NSPT group, supra and subgingival scaling and root planing was done at baseline followed by supra-gingival scaling and plaque control reinforcement two-weekly for one-month, and then monthly recall for supra-gingival scaling and plaque control reinforcement over entire duration of study. This is consistent with the study done by Badersten et al in 1984 who reported reduction of approximately $2 \mathrm{~mm}$ mean PPD with single supra and subgingival instrumentation followed by supra-gingival professional plaque control and no further improvement was achieved with repeated subgingival instrumentation. ${ }^{10}$ In our study, decrease in PPD was more at six months than three months which is in accordance with Badersten et $\mathrm{al}^{17}$ in which gradual decrease in PPD following root planing over a period of four to five months in 4-7 $\mathrm{mm}$ pocket depth or even more over nine months in deeper sites was seen. Following phase II and maintenance therapy, larger change may be due to increase in probing attachment and continued connective tissue reorganization or creeping attachment coronally. ${ }^{6}$ 
When comparing with NSPT group, the decrease in PPD $(0.17 \mathrm{~mm})$ and the gain in CAL $(0.06 \mathrm{~mm})$ were significantly higher after OFD from baseline to 6 months in our study. The findings are in accordance with the meta-analysis by Heitz-Mayfield et al (2002), in which at 12 months, open flap debridement resulted in slightly greater $(0.6 \mathrm{~mm})$ probing depth reduction and clinical attachment gain $(0.2 \mathrm{~mm})$ in deep pockets $\left(>6 \mathrm{~mm}\right.$ ) in non-furcation areas than SRP. ${ }^{18}$

In the NSPT group, the decrease of PPD from baseline to 6 months was $1.87 \mathrm{~mm}$ and the gain of CAL from baseline to six months was $1.00 \mathrm{~mm}$. Similarly, in the OFD group, the decrease of PPD from baseline to six months was $2.04 \mathrm{~mm}$ and the gain of CAL from baseline to six months was 1.06 $\mathrm{mm}$ are in agreement with Lindhe et al. (1982), also showed in SRP group (PPD >6 mm), the reduction in mean probing pocket depth was $2.6 \mathrm{~mm}$ in non-molar areas and $2.0 \mathrm{~mm}$ in molar areas and in the OFD group, the PPD reduction was $3.4 \mathrm{~mm}$ in non-molar areas and $2 \mathrm{~mm}$ in molar areas. In case of PPD $>6 \mathrm{~mm}$, the mean gain in CAL in SRP group were $0.9 \mathrm{~mm}$ in non-molar areas and $0.9 \mathrm{~mm}$ in molar areas and the mean gain in CAL in the OFD group was $1.5 \mathrm{~mm}$ in non-molar areas and $0.7 \mathrm{~mm}$ in molar areas. ${ }^{19}$ Considering various confounding factors like variation in sample size, inclusion and exclusion criteria, variation in methods and study designs, it is difficult to predict which factors have greater role in the decrease in probing pocket depth after periodontal therapies. Therefore, furthermore multicenter randomized controlled trials with greater sample size and longer duration will be needed to clarify the factors affecting the results of periodontal therapies.

\section{CONCLUSION}

The following conclusions were obtained from the present study:

- There was the significant improvement of periodontal status in terms of reduction in PPD, and gain in CAL from baseline to three and six months following nonsurgical and surgical periodontal therapy.

- The decrease in PPD was significantly more following surgical periodontal therapy than non-surgical periodontal therapy from baseline to three, and six months.

- The decrease in PPD was significantly higher at six months than three months in both the NSPT and OFD group.

- When comparing with the NSPT group, the gain in CAL was significantly higher in the OFD group at six months.

Thus, from the current study, authors would like recommend:

I. For decreasing of PPD, surgical therapy is preferred.

II. To obtain substantial and significant healing after periodontal therapy; post-treatment duration should be at least six months or even more with good plaque control.

\section{REFERENCES}

1. Flemming TF. Periodontitis. Ann Periodontol. 1999;4(1):32-7.

2. Petersen PE, Ogawa H. Strengthening the prevention of periodontal disease: the WHO approach. J Periodontol. 2005;76(12):2187-93.

3. van Palenstein Helderman W, Groeneveld A, Jan Truin G, Kumar Shrestha B, Bajracharya M, Stringer R. Analysis of epidemiological data on oral diseases in Nepal and the need for a national oral health survey. Int Dent J. 1998:56-61.

4. Graziani F, Karapetsa D, Alonso B, Herrera D. Nonsurgical and surgical treatment of periodontitis: how many options for one disease? Periodontol 2000. 2017;75(1):152-88.

5. Al-Shammari KF, Neiva RF,Hill RW, Wang HL. Surgical and non-surgical treatment of chronic periodontal disease. Int Chin J Dent 2002;2:15-32.

6. Kaldahl WB, Kalkwarf KL, Patil KD, Dyer JK, Dj P, Bates RE Jr. Evaluation of four modalities of periodontal therapy. Mean probing depth, probing attachment level and recession changes. J Periodontol. 1988 Dec;59(12)783-93.

7. Graziani F, Karapetsa D, Mardas N, Leow N, Donos N. Surgical treatment of the residual periodontal pocket. Periodontol 2000. 2018 Feb;76(1):150-63.

8. Kim TS, Schenk A, Lungeanu D, Reitmeir P, Eickholz P. Nonsurgical and surgical periodontal therapy in single-rooted teeth. Clin Oral Investig. 2007;11(4):391-9.

9. Haffajee AD, Cugini MA, Dibart S, Smith C, Kent RL, Socransky SS. Clinical and microbiological features of subjects with adult periodontitis who responded poorly to scaling and root planing. J Clin Periodontol. 1997 Oct 6;24(10):767-76.

10. Badersten A, Nilveus R, Egelberg J. Effect of nonsurgical periodontal therapy. III. Single versus repeated instrumentation. J Clin Periodontol. 1984 Feb; 11(2):114-24

11. Egelberg J. Current Facts on Periodontal Therapy Q \& A. Malmo, Sweden: OdontoScience; 1999. 32 p.

12. Rylander H LJ. Cause-related periodontal therapy. In: Lindhe J, Karring T, Lang NP, eds. Clin Periodontol Implant Dent 4th ed. Copenhagen, Denmark: Blackwell Munksgaard; 2004;432-43.

13. Walter B. Hall. Critical Decisions in Periodontology, Hamilton, BC Decker Inc. 2003. 324p.

14. Caton JG, Zander HA. The Attachment Between Tooth and Gingival Tissues After Periodic Root Planing and Soft Tissue Curettage. J Periodontol. 1979;50(9):462-6.

15. Morrison EC, Ramfjord SP, Hill RW. Short-term effects of initial, nonsurgical periodontal treatment (hygienic phase). J Clin Periodontol. 1980 Jun;7(3):199-211.

16. Ciancio SG. Non-surgical periodontal treatment. In: Proceedings of the World Workshop in Clinical Periodontics. Chicago: American Academy of Periodon- tology. 1989. II-4p.

17. Badersten A, Nilvéus R, Egelberg J. Effect of non-surgical periodontal therapy. J Clin Periodontol. 1981: Feb;8(1):57-72.

18. Heitz-Mayfield LJA, Trombelli L, Heitz F, Needleman I, Moles D. A systematic review of the effect of surgical debridement vs. nonsurgical debridement for the treatment of chronic periodontitis. J Clin Periodontol. 2002;29:92-102.

19. Lindhe J, Socransky SS, Nyman S, Haffajee A, Westfelt E. Critical probing depths in periodontal therapy. J Clin Periodontol. 1982 Jul;9(4):323-36. 